\title{
Isquemia miocárdica por consumo de dibutilona
}

\author{
Antonio Pascale* ${ }^{\star}$ Eleuterio Umpiérrez ${ }^{\dagger}$, Jorge Menonił, \\ Virginia Collazzi§, Virginia Rodríguez"
}

\section{Resumen}

Las catinonas sintéticas son drogas estimulantes de síntesis, comercializadas en el mercado ilegal por sus propiedades estimulantes y perturbadoras del sistema nervioso central, para su uso recreativo, solas, o como adulterantes de cocaína y derivados anfetamínicos. A nivel mundial existe un número creciente de reportes de intoxicaciones agudas y complicaciones por consumo de estas sustancias. En Uruguay fueron detectadas por primera vez en el año 2015, existiendo una información epidemiológica muy limitada. Las catinonas sintéticas no se detectan por las técnicas inmunocromatográficas disponibles en los laboratorios de los servicios de urgencias de nuestro país. Se describe el primer caso de intoxicación por una catinona sintética (dibutilona) en Uruguay, en un paciente de 45 años, por consumo de un polvo vendido como "éxtasis", presentando como complicación un síndrome coronario agudo, con una buena evolución posterior. La confirmación diagnóstica se realizó mediante su detección en orina por cromatografía de gases acoplada a espectrometría de masas. La estructura química de la dibutilona y su mecanismo de acción explican las manifestaciones clínicas y la complicación isquémica por vasoespasmo coronario. En ausencia de una noción clara de exposición y ante la situación epidemiológica actual, la presencia de un cuadro clínico compatible con una droga estimulante y resultado negativo para cocaína y anfetaminas en estudios inmunocromatográficos, debe hacer plantear la sospecha de otros estimulantes de síntesis como las catinonas, tal como ocurrió en este caso.

Palabras clave: Isquemia miocárdica

Drogas de diseño

Catinonas

Dibutilona

Intoxicación

Key words: $\quad$ Myocardial ischemia

Designer drugs

Cathinone

Dibutylone

Poisoning

\footnotetext{
* Médico toxicólogo. Profesor adjunto de Toxicología. Departamento de Emergencia, Hospital Británico. Montevideo, Uruguay. † Profesor adjunto de Química. Unidad de Medio Ambiente, Drogas y Doping. Instituto Polo Tecnológico de Pando, Facultad de Química, Universidad de la República, Canelones, Uruguay.

‡ Médico internista. Profesor adjunto de Clínica Médica 1. Departamento de Emergencia, Hospital Británico. Montevideo, Uruguay.

$\S$ Médica cardióloga. Departamento de Emergencia, Hospital Británico. Montevideo, Uruguay.

II Médica internista. Asistente de Clínica Médica 1. Departamento de Emergencia, Hospital Británico. Montevideo, Uruguay.

Correspondencia: Dr. Antonio Pascale.

Correo electrónico: antopascale@gmail.com

Los autores declaran no tener conflicto de intereis.

Recibido: $16 / 6 / 20$

Aprobado: $7 / 9 / 20$
} 


\section{Introducción}

Una nueva generación de feniletilaminas de síntesis ha emergido desde la primera década del siglo XXI y un significativo incremento de intoxicaciones agudas por catinonas sintéticas ha sido reportado a nivel mundial desde entonces ${ }^{(1)}$.

Las catinonas sintéticas se incluyen dentro de las denominadas drogas de síntesis o nuevas sustancias psicoactivas. Inicialmente vendidas como "sales de baño" o "euforizante legales", han sido ampliamente difundidas por internet con la aclaración de "no ser utilizadas para consumo humano", con el fin de evadir los controles legales. El origen de estos compuestos se halla naturalmente en la planta Khat (Catha edulis), conocida por efectos psicoestimulantes en África y en $\mathrm{Asia}^{(2)}$. Existe una gran variedad de catinonas sintéticas bajo el control de organismos regulatorios mundiales y de cada país o región. Desde el punto de vista químico, las catinonas sintéticas dentro del grupo de estimulantes son el grupo más grande de nuevas sustancias psicoactivas por su gran variedad. Esto se basa en que las catinonas son variaciones estructurales de las feniletilaminas en las cuales se coloca un grupo carbonilo en el carbono $\beta$ y por ende se llaman $\beta$-ceto feniletilaminas (figura 1). Las catinonas comparten mecanismos de acción tóxica como psicoestimulantes, pero éstos varían de acuerdo a su estructura. Sus efectos resultan, en mayor o menor medida, en estimulación dopaminérgica, noradrenérgica y sertoninérgica, con diferentes grados de potencia ${ }^{(2,3)}$.

Comparten similitud estructural con la anfetamina, el MDA (metilendioxianfetamina) o el MDMA (3,4 metilendioximetanfetamina) y mecanismos de acción similares, algunas con un perfil más estimulante y otras con un perfil más entactógeno o alucinógeno. A diciembre de 2019, más de 160 catinonas sintéticas habían sido reportadas al Sistema de Alerta Temprana de la Oficina de Naciones Unidas contra la Droga y el Delito (UNODC, por su sigla en inglés $)^{(4)}$. Actualmente muchos países han pasado a controlar estas sustancias por familias. Sin embargo, el comercio ilícito de estas sustancias persiste, solas o como adulterantes de otras drogas estimulantes, como cocaína o anfetaminas ${ }^{(2-4)}$.

Se consumen bajo forma de polvo o "cristales", por vía inhalada (esnifada) o por vía oral. La vía intraveno$\mathrm{sa}$, intramuscular o rectal son mucho menos frecuentes como forma de consumo ${ }^{(2,5)}$. Se han encontrado en tabletas o "pastillas", adulterando o sustituyendo drogas vendidas como "éxtasis" (3,4 metilendioximetanfetamina o MDMA $)^{(6)}$. Las adulteraciones de pastillas o cristales de "éxtasis" con catinonas incrementan significativamente el riesgo de complicaciones agudas. Es frecuente el policonsumo con alcohol y otras drogas estimulantes, potenciando su toxicidad aguda ${ }^{(1-3,5)}$.
La Asociación de Centros de Toxicología de Estados Unidos reportó los primeros casos de intoxicaciones agudas por estas sustancias en el año $2010^{(2)}$. Dependiendo de la sustancia consumida, la intoxicación aguda se caracteriza por complicaciones tales como alteraciones neuropsiquiátricas, así como elementos clínicos de un síndrome simpaticomimético o serotoninérgico, o $\operatorname{ambos}^{(1,2,5,7)}$ (tablas 1 y 2). Es frecuente el policonsumo (asociación con otras drogas), agravando la toxicidad aguda por estas sustancias.

La dibutilona (también denominada bk-DMBDB) es la 1-(1,3-benzodioxol-5-il)-2-(dimetilamino)-butan-1-ona, análogo de la N-metil-1,3-metilendioxifenil-2-butanamina con un grupo ceto en posición 1 (MBDB $)^{(8)}$. La butilona, dibutilona y otras beta-ceto-metilendioxianfetaminas evidenciaron efectos estimulantes y entactógenos, por lo que son vendidas frecuentemente como "éxtasis", en tabletas ("pastillas") o polvo ("cristales") $)^{(9)}$. Existe información toxicológica limitada en relación con los aspectos toxicocinéticos y a la intoxicación aguda por dibutilona ${ }^{(8-11)}$.

En América del Sur existió una primera alerta sobre la presencia de tabletas con butilona en Colombia, en el año $2017^{(12)}$. En nuestro país, las catinonas fueron detectadas en orina de asistentes a fiestas electrónicas en el año $2015^{(13)}$. La dibutilona fue detectada en orina de la misma forma por primera vez en Uruguay en $2017^{(14)}$. En nuestro país no existen registros de prevalencia de consumo de estas sustancias con fines recreativos. Debido a las dificultades de detección de catinonas por métodos de inmunoensayo utilizados para "drogas clásicas" en servicios de urgencia y emergencia, se hace difícil contar con un registro de intoxicaciones agudas por estas sustancias en nuestro medio.

\section{Objetivo}

Reportar una intoxicación aguda por un polvo vendido como "éxtasis", presentando como complicación un síndrome coronario agudo, con un estudio toxicológico analítico que evidencia la presencia de dibutilona, en ausencia de MDMA y de otros estimulantes. Se trata del primer caso reportado de intoxicación por dibutilona en nuestro país.

\section{Caso clínico}

Paciente de 45 años, sexo masculino, deportista, sin antecedentes de consumo de sustancias psicoactivas ni otros antecedentes personales a destacar. Consulta en departamento de emergencia por un cuadro clínico caracterizado por malestar general, vómitos reiterados, epigastralgia y dolor en hemiabdomen superior de difícil caracterización, que aparece cinco horas después del consumo, durante varias horas, de dosis desconocida de 


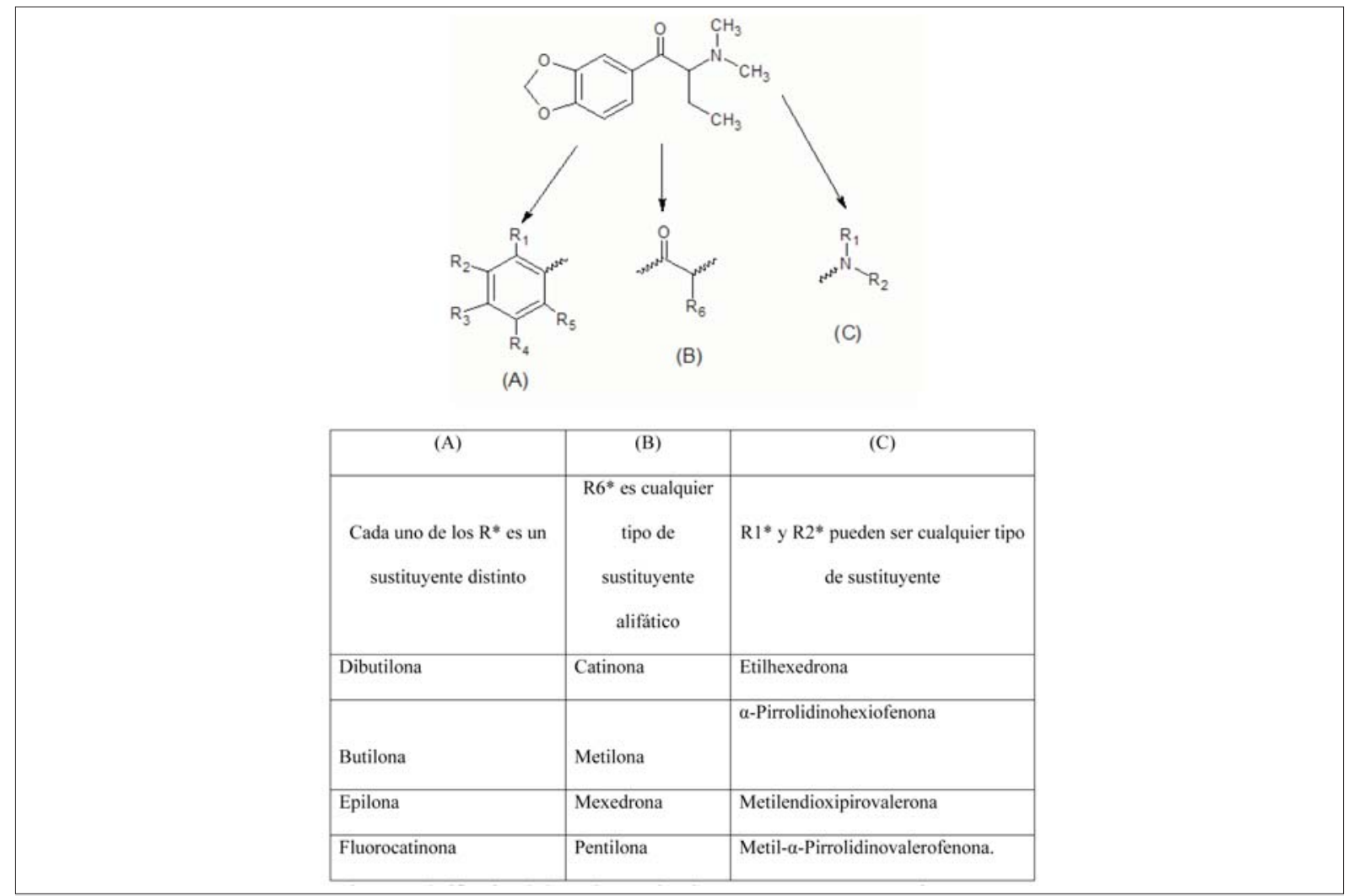

Figura 1. Clasificación de las catinonas sintéticas según su estructura química. * $R$ : se refiere a sustituyente o grupo sustituyente.

un polvo vendido como "cristales" o "éxtasis", diluido en un litro de champán y asociado a la ingesta de un comprimido de $20 \mathrm{mg}$ de tadalafilo. Dicho consumo se inició 24 horas antes de la consulta. Niega disnea, palpitaciones y síncope. Al ingreso paciente lúcido, GCS (Glasgow Coma Scale) 15, eupneico, frecuencia cardíaca de $80 \mathrm{cpm}$, presión arterial 160/90 mmHg y temperatura axilar de $36,5^{\circ} \mathrm{C}$. Pupilas intermedias, tono muscular normal, sin movimientos anormales.

Electrocardiograma (ECG) inicial de 12 derivaciones evidenció una inversión de la onda $\mathrm{T}$ en derivadas DII, DIII y AVF con segmento ST isoeléctrico compatible con isquemia miocárdica de cara inferior. Se administró una dosis de diazepam de $10 \mathrm{mg}$.

Un segundo ECG mostró la misma alteración 6 horas después. Los niveles de troponina I fueron de 0,005 $\mathrm{ng} / \mathrm{mL}$ al ingreso, 6 horas y 12 horas después. El dolor y los síntomas digestivos mejoraron en las primeras 6 horas. Los estudios de laboratorio evidenciaron creatin-fosfokinasa (CPK) total de $187 \mathrm{U} / 1$, creatininemia de $1,12 \mathrm{mg} / \mathrm{dl}$ y azoemia de $0,30 \mathrm{~g} / \mathrm{l}$. El funcional y enzimograma hepático, ionograma y amilasemia fueron normales. Ecografía abdominal sin alteraciones. La determinación de drogas en orina por inmunocromatografía fue negativa para benzoilecgonina (metabolito inactivo de la cocaína), anfetamina, metanfetamina and MDMA (3,4-metilendioximetanfetamina) al ingreso. Se solicitó un análisis por cromatografía de gases acoplada a espectrometría de masas (GC/MS) de la muestra de orina, pero el resultado del estudio iba a obtenerse a las 72 horas.

El paciente fue ingresado para observación clínica y estudios, con diagnóstico de síndrome coronario agudo sin elevación del segmento ST, secundario a vasoespasmo coronario.

Permaneció asintomático en evolución. La normalización de las alteraciones en la onda T ocurrió 36 horas después de su admisión. El ecocardiograma transtorácico evidenció una leve hipertrofia ventricular izquierda, con contractilidad conservada y función sistólica normal (FEVI 65\%). Una prueba ergométrica de esfuerzo 72 horas luego del ingreso resultó submáxima, suficiente por frecuencia, sin ángor ni signos de isquemia.

Se decide, en conjunto con el paciente, dada la buena evolución clínica y los estudios mencionados, no realizar en esta oportunidad una cineangiocoronariografía (CACG), quedando supeditada a la evolución clínica. Tampoco contamos con un estudio funcional de alta 
Tabla 1. Manifestaciones clínicas y complicaciones reportadas en intoxicaciones agudas por catinonas sintéticas (adaptado de Prosser et al, 2012).

\begin{tabular}{ll}
\hline Neurológicas & $\begin{array}{l}\text { Cefaleas, distonías, hiperreflexia, } \\
\text { parestesias, temblores, mioclonias, } \\
\text { convulsiones. }\end{array}$ \\
\hline Neuropsiquiátricas & $\begin{array}{l}\text { Ansiedad, agitación, confusión, } \\
\text { alucinaciones, ideación paranoide, } \\
\text { psicosis aguda. }\end{array}$ \\
\hline Cardiovasculares & $\begin{array}{l}\text { Dolor torácico (precordial, retroesternal), } \\
\text { palpitaciones, taquicardia, hipertensión } \\
\text { arterial, miocarditis. }\end{array}$ \\
\hline Digestivas & $\begin{array}{l}\text { Náuseas, dolor abdominal, elevación de } \\
\text { transaminasas hepáticas. }\end{array}$ \\
\hline Musculo-esqueléticas & $\begin{array}{l}\text { Elevación de creatinfosfokinasa, } \\
\text { rabdomiólisis. }\end{array}$ \\
\hline Renales & Injuria renal aguda. \\
\hline Oftalmológicas & Midriasis, alteración de la visión. \\
\hline Respiratorias & Disnea, polipnea. \\
\hline Otras & Hipertermia, sudoración, hiponatremia. \\
\hline
\end{tabular}

sensibilidad diagnóstica, como un centellograma miocárdico o ecocardiograma estrés.

El estudio por cromatografía de gases/espectrometría de masas (GC/MS) reveló la presencia de dibutilona (bk-DMBDB) y el paciente fue dado de alta.

Un monitoreo ambulatorio de la presión arterial (MAPA), realizado luego de un mes, evidenció cifras elevadas de presión arterial, interpretada como hipertensión arterial esencial. El diagnóstico final fue síndrome coronario agudo, sin elevación del segmento ST, por la catinona sintética hallada, planteándose hipótesis en la fisiopatología del desarrollo de esta complicación, con limitaciones por los estudios realizados, como probable vasoespasmo coronario o aumento de la demanda miocárdica de oxígeno en el curso de la intoxicación aguda, sin poder descartar la existencia de coronariopatía.

\section{Discusión}

El paciente presentó síntomas inespecíficos reportados luego del consumo de catinonas (malestar general, vómitos, dolor abdominal e isquemia miocárdica) $)^{(1,2,5)}$. Las cifras elevadas de presión arterial al ingreso pueden resultar del efecto simpaticomimético, si bien luego, en la evolución, el hallazgo de cifras tensionales elevadas fuera del episodio agudo y la hipertrofia ventricular izquierda permiten plantear una hipertensión arterial
Tabla 2. Síndromes tóxicos reportados en intoxicaciones agudas por drogas estimulantes como catinonas sintéticas.

\begin{tabular}{ll}
\hline Sindrome simpaticomimético & \multicolumn{1}{c}{ Sindrome serotoninérgico } \\
\hline - Midriasis & - Trastornos de conciencia: \\
- Taquicardia & excitación, confusión. \\
- Hipertensión arterial & - Trastornos de la actividad \\
- Arritmias cardíacas & muscular: rigidez, mioclonias, \\
- Dolor torácico (isquemia & trismus, convulsiones. \\
miocárdica por vasoespasmo & - Disautonomías: hipertermia, \\
coronario) & cambios en la frecuencia cardíaca \\
- Infartos o hemorragias & y la presión arterial, sialorrea, \\
cerebrales (por vasoespasmo e & vómitos y diarrea, incontinencia \\
injuria endotelial). & urinaria \\
- Temblor & \\
- Convulsiones & \\
\hline
\end{tabular}

esencial de base. La ausencia de otros elementos clínicos de estimulación simpática (midriasis, taquicardia) podrían explicarse por la consulta tardía y por un perfil psicoactivo MDMA símil en una primera fase de la intoxicación aguda ${ }^{(15,16)}$. La dibutilona se metaboliza a butilona $^{(11)}$. Si bien la información relacionada a la vida media de eliminación de ambas sustancias es muy limitada, las catinonas más estudiadas tienen una vida media corta, oscilando entre 1,5 y 2,5 horas, lo que podría también explicar la ausencia de otros elementos clínicos de estimulación simpática o sertoninérgica, o ambas ${ }^{(17)}$, y evidenciar la complicación secundaria al consumo, como ocurrió en este caso.

Se ha reportado un incremento de los niveles de serotonina, dopamina y noradrenalina luego del consumo de butilona, compuesto con estructura similar a la dibutilona. Si bien un caso letal de síndrome serotoninérgico fue descrito ${ }^{(9)}$, la dibutilona es considerada un inhibidor no selectivo de la recaptación de monoaminas endógenas (dopamina, noradrenalina), similar a la cocaína, con una afinidad cinco veces mayor por el transportador dopaminérgico y noradrenérgico que por el transportador serotoninérgico ${ }^{(16)}$. Esto explicaría en este caso la ausencia de elementos serotoninérgicos característicos y otras complicaciones agudas (neuropsiquátricas, rabdomiólisis, injuria renal aguda, entre otras). La hipótesis fisiopatológica de vasoespasmo coronario podría ser explicada por la estimulación alfa-adrenérgica secundaria al aumento de los niveles de noradrenalina. Este mecanismo fisiopatológico, junto con el aumento del consumo de oxígeno con disbalance oferta/demanda, podría explicar el síndrome coronario agudo planteado, la normalización de las alteraciones electrocardiográficas y una prueba ergométrica negativa para isquemia a los tres días del consumo. Es de destacar como limitación en es- 
te caso que la confirmación de vasoespasmo coronario se realiza mediante estudios invasivos del tipo de la CACG, donde se demuestra la ausencia de lesiones coronarias angiográficamente significativas y se puede demostrar el vasoespasmo a través de estimulación con drogas específicas intracoronarias (acetil colina - ergonovina). El test funcional sin evidencia de isquemia realizado al paciente en la evolución, no descarta la existencia de lesiones coronarias que eventualmente podría presentar, teniendo en cuenta la sensibilidad y especificidad de este estudio: $67 \%$ y $72 \%$ respectivamente. Lo analizado previamente representa una limitación en la hipótesis fisiopatológica planteada, no pudiendo descartar patología coronaria previa en un paciente de 45 años con diagnóstico de hipertensión arterial, con o sin vasoespasmo.

Al igual que ocurre con otras drogas estimulantes, no existe un tratamiento antidótico en caso de intoxicación aguda por catinonas sintéticas. El tratamiento de la complicación se basa en la administración de benzodiacepinas con el fin de reducir el tono simpático central. Los betabloqueantes están contraindicados dado que al liberar el efecto alfa-adrenérgico puede agravar la isquemia miocárdica. No se encontraron interacciones entre las catinonas y el uso de tadalafilo, así como tampoco posibles efectos adversos cardiovasculares de tadalafilo en el contexto de este cuadro clínico ${ }^{(18)}$.

Existen vías metabólicas comunes entre la dibutilona y otras catinonas, así como otros estimulantes de tipo anfetamínico. La asociación con alcohol etílico podría agravar la toxicidad de la dibutilona incrementando su biodisponibilidad $^{(1,8,15,19)}$.

Este es el primer caso reportado de ángor e isquemia miocárdica transitoria por el consumo de dibutilona. Futuras investigaciones son necesarias para evaluar la cardiotoxicidad de dibutilona y butilona. Las adulteraciones con catinonas reportadas en nuestro país deben tenerse presente en los planteos diagnósticos etiológicos de los cuadros de sobredosis por drogas estimulantes, fundamentalmente en aquellos casos de noción de consumo de "éxtasis" en "pastillas" o "cristales" con complicaciones agudas resultantes en una mayor estimulación simpática, así como el resultado de detecciones negativas en orina de MDMA o anfetaminas por técnicas inmunocromatográficas o de screening, de creciente uso en los servicios de urgencias y emergencias.

\section{Summary}

Synthetic cathinone are stimulating synthetic drugs used for recreational purposes on their own or as adulterants in cocaine and amphetamines derivatives that are traded in illegal markets given their stimulating and disturbing properties on the central nervous system.
Globally, there is a growing number of acute intoxications and complications as a consequence of consumption of these substances. In Uruguay they were first detected in 2015, there being limited epidemiological data. Synthetic cathinone are not detected by the immunochromatographic tests available at the laboratories of the emergency services in our country. The study describes the first case of intoxication by a synthetic cathinone (dibutylone) in Uruugay, in a 45 year old patient as a result of powder sold as "ecstasy". The patient presented an acute coronary syndrome, subsequent evolution being good. Diagnostic confirmation was made in urine using gas chromatography coupled with mass spectrometry. The chemical structure of dibutylone and its action mechanism explain the clinical manifestations and the coronary vasospasm causing ischemia. When there is no clear sign of a patient having been exposed to this substance and upon the current epidemiological state, the presence of clinical symptoms that are compatible with a stimulating drug and a negative result for cocaine and amphetamines in immunochromatographic tests must lead us to be suspicious about the presence of synthetic stimulating substances as cathinone, as in the case studied.

\section{Resumo}

Catinonas sintéticas são drogas estimulantes sintéticas, comercializadas no mercado ilegal por suas propriedades estimulantes e perturbadoras do sistema nervoso central, para uso recreativo isoladamente ou como adulterantes da cocaína e derivados de anfetaminas. Em todo o mundo cresce o número de relatos de intoxicações agudas e complicações decorrentes do uso dessas substâncias. No Uruguai, foram detectados pela primeira vez em 2015 com informações epidemiológicas muito limitadas. As catinonas sintéticas não são detectadas pelas técnicas imunocromatográficas disponíveis nos laboratórios de pronto-socorro de nosso país. Descreve-se o primeiro caso de intoxicação por catinona sintética (dibutilona) em nosso país, em um paciente de 45 anos, devido ao consumo de um pó vendido como "ecstasy", apresentando como complicação uma síndrome coronariana aguda, com boa evolução posterior. A confirmação diagnóstica foi feita por sua detecção na urina por cromatografia gasosa acoplada à espectrometria de massa. A estrutura química da dibutilona e seu mecanismo de ação explicam as manifestações clínicas e complicações isquêmicas devido ao vasoespasmo coronariano. Na ausência de uma noção clara de exposição e dada a situação epidemiológica atual, a presença de quadro clínico compatível com droga estimulante e resultado negativo para cocaína e anfetaminas em estudos imunocromatográficos, deve levantar a suspeita de ou- 
tros estimulantes sintéticos como as catinonas, como aconteceu neste caso.

\section{Bibliografía}

1. Prosser JM, Nelson LS. The toxicology of bath salts: a review of synthetic cathinones. J Med Toxicol 2012; 8:33-42.

2. Nelson ME, Bryant SM, Aks SE. Emerging drugs of abuse. Emerg Med Clin North Am 2014; 32:1-28.

3. Baumann MH, Solis E Jr, Watterson LR, Marusich JA, Fantegrossi WE, Wiley JL. Baths salts, spice, and related designer drugs: the science behind the headlines. J Neurosci 2014; 34(46):15150-8.

4. United Nations Office on Drugs Crime. Laboratory and Scientific Section Portals. Synthetic cathinones. Disponible en: https://www.unodc.org/LSS/SubstanceGroup/Details/67b1ba69-1253-4ae9-bd93-fed1ae8e6802 [Consulta: 31 mayo 2020].

5. Banks ML, Worst TJ, Rusyniak DE, Sprague JE. Synthetic cathinones ("bath salts"). J Emerg Med 2014; 46(5):632-42.

6. Oliver CF, Palamar JJ, Salomone A, Simmons SJ, Philogene- Khalid H, Stokes-McCloskey N, et al. Synthetic cathinone adulteration of illegal drugs. Psychopharmacology (Berl) 2019; 236(3):869-79.

7. Derungs A, Schietzel S, Meyer MR, Maurer HH, Krähenbühl S, Liechti ME. Sympathomimetic toxicity in a case of analytically confirmed recreational use of naphyrone (naphthylpyrovalerone). Clin Toxicol (Phila) 2011; 49(7): 691-3.

8. Krotulski AJ, Mohr AL, Papsun DM, Logan BK. Dibutylone (bk-DMBDB): intoxications, quantitative confirmations and metabolism in authentic biological specimens. J Anal Toxicol 2018; 42(7):437-45.

9. Warrick BJ, Wilson J, Hedge M, Freeman S, Leonard K, Aaron C. Lethal serotonin syndrome after methylone and butylone ingestion. J Med Toxicol 2012; 8:65-8.

10. Rojek S, K³y M, Strona M, Maciów M, Kula K. "Legal highs"- toxicity in the clinical and medico-legal aspect as exemplified by suicide with bk-MBDB administration. Forensic Sci Int 2012; 222(1-3):e1-6.

11. Zaitsu K, Katagi M, Kamata HT, Kamata T, Shima N, Miki A, et al. Determination of the metabolites of the new designer drugs bk-MBDB and bk-MDEA in human urine. Forensic Sci Int 2009; 188(1-3):131-9.

12. Observatorio de Drogas de Colombia. Detección de nueva catinona sintética: comprimidos en forma de Hello Kitty con contenido de butilona. Agosto 2017. Disponible: http://www.cicad.oas.org/oid/sata/colombia/SAT_sobre_Drogas_de_Colombia_alerta_butilona.pdf[Consulta: 15 agosto 2018].

13. Junta Nacional de Drogas. Observatorio Uruguayo de Drogas; Umpierrez E. Screening de nuevas sustancias psicoactivas y cocaína en muestras de orina en fiestas electrónicas en Uruguay. 2016. Disponible en: https://www.gub.uy/ junta-nacional-drogas/comunicacion/publicaciones/screening-nuevas-sustancias-psicoactivas-cocaina-muestras-orina-fiestas [Consulta: 31 mayo 2020].

14. Junta Nacional de Drogas. Observatorio Uruguayo de Drogas; Umpierrez E. Informe Final: screening de nuevas sustancias psicoactivas, THC y cocaiìna en muestras de orina obtenidas en una fiesta musical en el aìrea metropolitana. 2017. Disponible en https://www.gub.uy/junta-nacional-drogas/comunicacion/publicaciones/nuevas-sustancias-psicoactivas-otras-drogas-abuso-consumidas-fiestas [Consulta: 31 mayo 2020].

15. Krotulski AJ, Mohr AL, Fogarty MF, Logan BK. The detection of novel stimulants in oral fluid from users reporting ecstasy, molly and MDMA ingestion. J Anal Toxicol 2018; 42(8):544-53.

16. Simmler LD, TA Buser TA, Donzelli M, Schramm Y, Dieu LH, Huwyler J, et al. Pharmacological characterization of designer cathinones in vitro. Br J Pharmacol 2013; 168:458-70.

17. Burillo-Putze G, Díaz BC, Pazos JL, Mas PM, Mirò̀ O, Puiguriguer P, et al. Drogas emergentes (I): smart drugs. An Sist Sanit Navar 2011; 34(2):263-74.

18. Kloner RA, Mitchell M, Emmick JT. Cardiovascular effects of tadalafil. Am J Cardiol 2003; 92(9A):37M-46M.

19. Papaseit E, Pérez-Mañá C, de Sousa Fernandes Perna EB, Olesti E, Mateus J, Kuypers KP, et al. Mephedrone and alcohol interactions in humans. Front Pharmacol 2020; $10: 1588$

\section{Contribución de autores}

Todos los autores participaron en la concepción, diseño, discusión y análisis del caso clínico presentado. El Dr. Antonio Pascale y Prof. Adj. Eleuterio Umpiérrez aportaron elementos teóricos inherentes a la toxicología y química de las catinonas sintéticas. La Dra. Virginia Rodríguez contribuyó además con una revisión bibliográfica inicial relacionada a la temática. El Dr. Jorge Menoni y la Dra. Virginia Collazi contribuyeron al enfoque médico-cardiológico de la discusión. Todos los autores participaron de la búsqueda bibliográfica. Antonio Pascale, https://orcid.org/0000-0002-8553-1323

Eleuterio Umpierrez, https://orcid.org/0000-0002-4863-3283

Jorge Menoni, https://orcid.org/0000-0002-0620-8692

Virginia Collazzi, https://orcid.org/0000-0001-7714-8052

Virginia Rodríguez, https://orcid.org/0000-0001-8367-0808 